\title{
Intelligent Digital Twin to make Robot Learn the Assembly process through Deep Learning
}

\author{
Bilal Ahmad ${ }^{1}$, Mian Usman Sattar ${ }^{2}$, Hamza Wazir Khan ${ }^{3}$, Zarmeen Qureshi ${ }^{4}$, Raza Hasan ${ }^{5}$ Ali Ahmad ${ }^{6}$, \\ Moeen-ud-Din Azad ${ }^{7}$ \\ 1,3,7 School of Business and Economics, University of Management and Technology Lahore, Pakistan. \\ ${ }^{2,4}$ Department of Management Sciences, Beaconhouse National University, Pakistan. \\ ${ }^{5}$ Middle East College, Oman. \\ ${ }^{6}$ University of Southern Denmark. \\ Email: 2604bilal@gmail.com
}

(Received: 22 July 2021; Accepted:01 Sep 2021; Issue Published: 12 Sep 2021)

\begin{abstract}
The objective of this paper is to utilize deep learning technology to develop an intelligent digital twin for the operational support of a human-robot assembly station. Digital twin, as a virtual portrayal, is used to design, simulate, and optimize the complexity of the assembly system. For testing purposes, a convolutional neural network (CNN) is integrated with a digital twin. It is used for the application of a collaborative robot for an assembly application. Collaborative robots are a new form of industrial robots that are safe for humans and can work alongside humans and have received ample attraction in recent years for automation of simple to complex tasks.
\end{abstract}

KEYWORDS: Digital Twin, Deep Learning, Robots, Collaborative Robots, Complex Tasks.

\section{INTRODUCTION}

Assembly accounts for a considerable proportion of production activities [1]. It is the integration of parts and components into working products for end-users. It has often been difficult to use automation solutions in assembly lines because assembly often involves handling of large number and variety of parts [2]. Also, with different product variants, the shape, design, and joining process vary, contributing to the total complexity of the process. And therefore, assembly is often considered a difficult process for automation [3]. An example of this is smartphone assembly, where different variants require the products to be assembled manually.

As a result, $90 \%$ of smartphone assembly is manual. The result of manual assembly is that human workers must repeatedly perform repetitive and boring tasks, unhealthy and unsafe postures, many errors, and defective products (the defective products are discovered late during production and require costly rework).

It is also aligned with the Lean manufacturing viewpoint, which has been dominant in the manufacturing landscape since the 1950s. Lean automation emphasis avoiding wastes and close integration of humans in the production process. Lean manufacturing also highlights a concept of hybrid automation by merging human flexibility and machine efficiency [4]. However, conventionally it was difficult to achieve as conventional robots were unsafe for humans and were placed in close fencing in factories.

Telecommunications companies have grown considerably when customers play a crucial role in ensuring consistent returns and thus customers are solemnly taken into account. The inclination of clients to go towards other networks that are desirable varies for many reasons such as price, call quality, services billing, and network accessibility [4].

The new generation of industrial robots are safe for humans and are light weighted. They can be used to introduce flexible human-robot assembly stations.

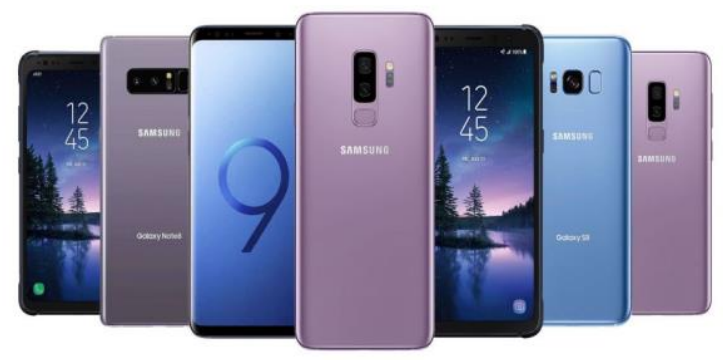

Figure 1: An example of Samsung phones with various variants and frequent new models

Due to frequent changes, the design, development, and operation of human-robot assembly systems require new and innovative approaches for design and validation. There are difficulties in several phases of 
the production framework plan, improvement, and activity, for example, to structure the human-robot communication (HRC) framework, protection analysis of the collaborating human, collision detection, robot programming, and managing this as often as possible at whatever point a change is experienced in production parameters. (such as a new batch of production).

However, in assembly, as already said, the complexity is high and new methods of design and control of these human-robot systems are needed [5]. The digital twin is a new concept of designing and controlling a complex physical system from digital simulation[6].

However, the idea of integrating artificial intelligence into a simulation-based digital twin is new and exciting for the manufacturing community. The example used to validate the study is LEGO construction bricks.

\section{LITERATURE REVIEW}

\subsection{Technologies to Explore}

The main aim is to simulate in parallel but running faster than the real system. A nonconformity from the real system can initiate the solution to the multifaceted system. This project enables us to explore the following technologies [7-8]:

TABLE 1. Technologies to explore

\begin{tabular}{|l|l|l|}
\hline Technology & Tool & Hardware \\
\hline Deep learning & $\begin{array}{l}\text { Convolutional } \\
\text { neural network }\end{array}$ & $\begin{array}{l}\text { Window- } \\
\text { based PC }\end{array}$ \\
$\begin{array}{l}\text { Continuous } \\
\text { Simulation }\end{array}$ & $\begin{array}{l}\text { Process } \\
\text { simulate }\end{array}$ & $\begin{array}{l}\text { Window- } \\
\text { based PC }\end{array}$ \\
\hline $\begin{array}{l}\text { Human-robot } \\
\text { interaction }\end{array}$ & $\begin{array}{l}\text { A collaborative } \\
\text { robot } \\
\text { Robot virtual } \\
\text { controller) }\end{array}$ & $\begin{array}{l}\text { Linux based } \\
\text { PC }\end{array}$ \\
\hline
\end{tabular}

The LEGO classification model built through deep learning uses a convolutional neural network based on Windows. A digital twin of a collaborative robot can be developed in Techniques used for Preprocessing software, which is also Windows operating system.

The interaction between human-robot, which can be exploited as a virtual robot controller, is based on the Linux operating system.

\subsection{Convolutional Neural Network}

The science of convolutional neural networks is used to classify images of data set, which is an extended branch of deep learning, relevant for its application in image recognition and classification of imagery data sets [7]. The usability of already presented engineering practices has appeared to be validated because structuring a CNN design may become a tiring and hectic task. Accordingly, unique existing systems were tried to locate the best reasonable [8]. The attractive element of CNN is its ability to misuse spatial or transitory information relationships. The geography of $\mathrm{CNN}$ is divided into several phases in which convolutions, non-direct handling units, and subexamining layers are combined.

\subsection{Techniques Used for Preprocessing}

Different libraries give usefulness to play out this assignment. Various calculations are applied to the image-based data set by using these libraries, thus improving the readability of auxiliary data. The techniques used in this study are presented in the following [9]:

$$
\begin{aligned}
& \text { i. Data Augmentation } \\
& \text { ii. Rescaling of Images }
\end{aligned}
$$

\section{PROPOSED METHODOLOGY}

\subsection{Data Set}

LEGO data is obtained from the Kaggle, which is open-source data. There are 6379 RGBA images of size 200,200 pixels. Images are distributed into 16 classes.
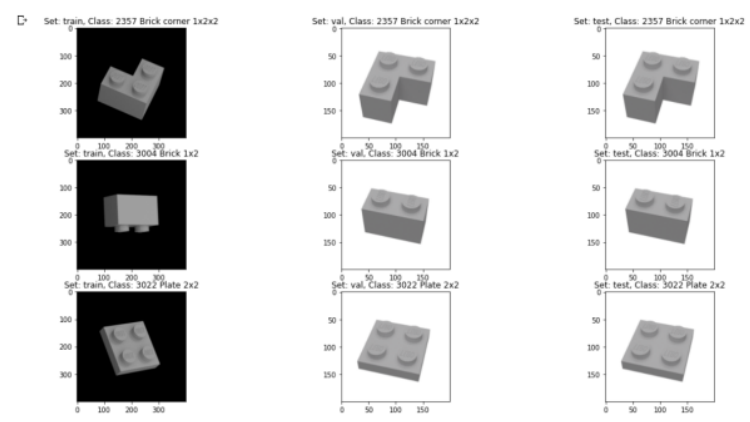

Figure 2: Images of the Dataset 


\subsection{Model Architecture}

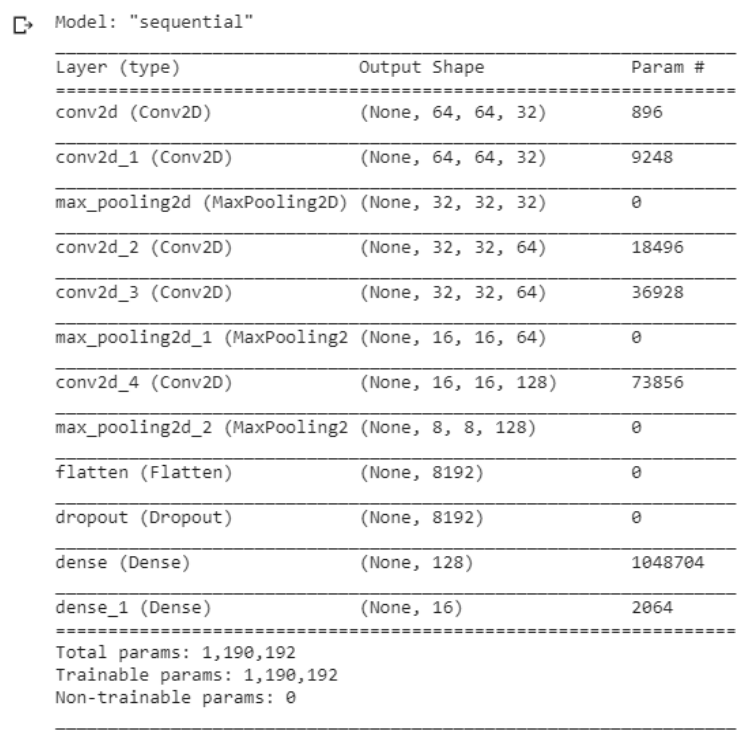

Figure 3: Trainable parameters

Several architectures are defined/ introduced in a convolutional neural network like Lenet, VGG, Google net, etc. For this study, I used Residual network (ResNet), an award winner in 2015 [9]. By using ResNet we were able to train a model of layers of 150 plus. Before this, it was hard to train a model of such a number of layers because of a vanishing gradient that at some point was unable to train the model.

In ResNet, it is possible because of the skip connections between the hidden layers of the model [10]. The ResNet50 model was used for training, which is based on CNN and SVM Classifiers for feature extracting from the dataset. [11].

A sequential model is designed and used for the recognition of Lego pieces. The model is the 4 Conv layers model. The kernel size of 3 is used in the model, whereas the stride and significant labor are still needed when a solution to the maxpool size is equal to 2 , and with this, the padding is equal to valid.

In the CNN model, the parameters of a neural network are the weights of the connections of the neurons among the layers. In this case, these parameters are learned during the training stage. Total trainable parameters available in the model are 1,190,192, whereas the non-trainable parameters are zero.

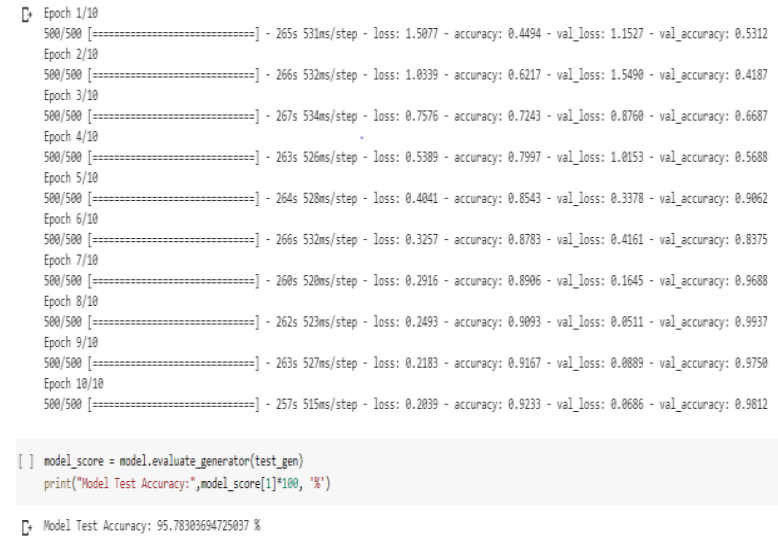

Figure 4: Accuracy Result

After training data at 10 epochs model can give an accuracy of 92.3 percent and at the testing data set the model give an accuracy of 95.78 percent.

\subsection{Collaborative Robots}

Collaborative robots are a new advanced form of industrial robots that are harmless for humans and can work together with humans. Collaborative robot's conception was introduced as an intelligent assist device to support and help human workers[9]. The robot as a supporting device was able to manipulate objects that were ergonomically challenging for
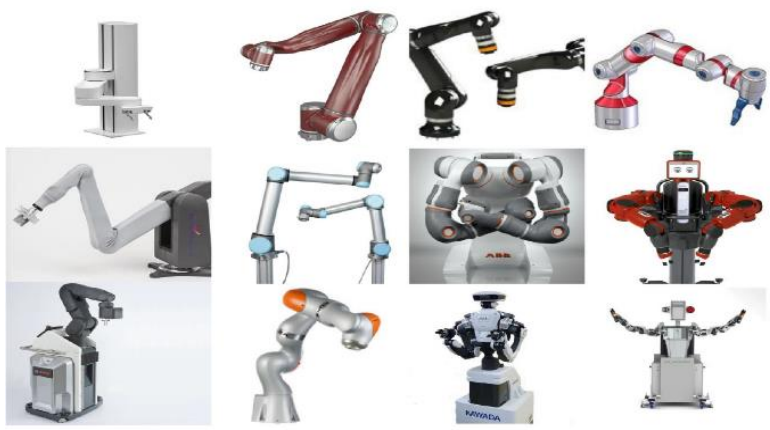

Figure 5: Various off-the-shelf collaborative robots

human workers. The unique or differentiating features of collaborative robots compared to classical industrial robots are their ability to safely interact with fellow humans[10]. Although the concept of collaborative robots has been explored in various domains (Figure) such as hospitals, hotels, operation theaters but an ample attraction is received from the manufacturing community. When used in manufacturing processes, the benefits of collaborative robots are expected to be increased productivity, improved ergonomics, and increased quality of the produced products. 
While the application of cobot in most industrial applications is still difficult to be described as collaborative.

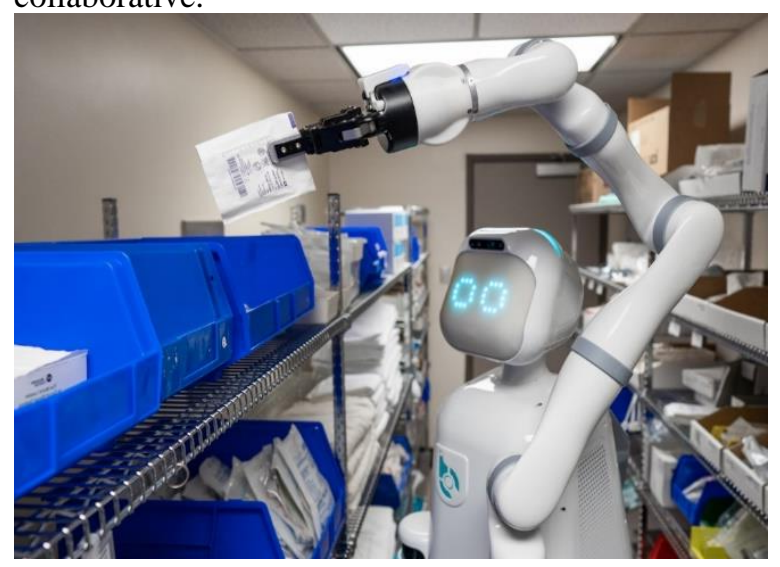

Figure 6: A human-friendly robot used in a hospital (Google search)

The resulting applications often do not generate much value in terms of flexibility and productivity, while their interaction with humans remains in question [3].

\section{RESULTS AND DISCUSSION}

\subsection{Simulation and Digital Twin}

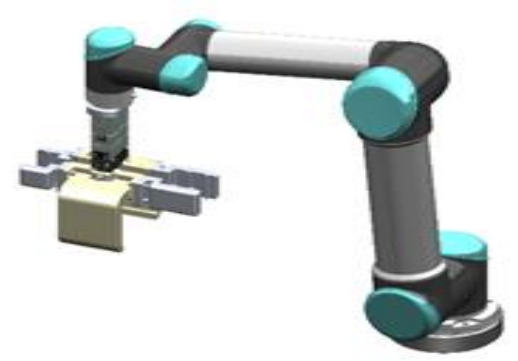

Figure 7: Stimulation Model

It is the digital and dynamic counterpart of the physical system and is created in Tecnomatix software. It is a 3-dimensional accurate model of all the elements of the physical system [15].

The 3D elements or geometrical elements are created in SolidWorks software and are then imported into the Tecnomatix software. Tecnomatix software imports JT (Jupiter Tessellation) format, a Computer-Aided Design (CAD) data format for data integration between different CAD platforms.

The evaluation is done manually in DT-Design. However, the DT-Execution synchronizes real-world data with the DT and may automate the evaluation cycle [13-14].

Significant labor is still needed when a solution to the given topic is developed and presented before it can be implemented to the human user.

\subsection{Tecnomatix Process Simulate}

After classifying the model through a convolutional neural network. The next phase was to create the simulation of the assembly station in software. The software used for the purpose of the simulation was Tecnomatix Process Simulate by Siemens. Tecnomatix is a comprehensive process modeling software in the PLM package of SIEMENS. The software can model robotic actions, human digital modeling, and production tasks in an integrated environment [16]. Various types of industrial robots can be modeled in the software given their torque and speed limits at various degrees of freedom (DOF).

\subsection{Data Connected Simulation (The Digital Twin Model)}

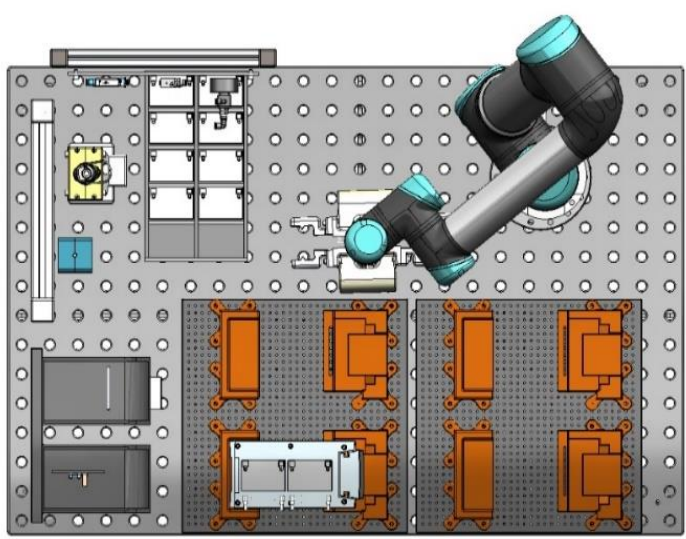

Figure 8: The 3-dimensional simulation mode

It is the digital and dynamic counterpart of the physical system and is created in Tecnomatix software. It is a 3-dimensional accurate model of all the elements of the physical system[12]. The 3D elements or geometrical elements are created in SolidWorks software and are then imported into the Tecnomatix software. Tecnomatix software imports JT (Jupiter Tessellation) format, a Computer-Aided Design (CAD) data format for data integration between different CAD platforms[17].

\subsection{Test and Analysis}

The results achieved in the machine learning stage are then integrated with the simulation model. The simulation is able to import data from CSV spreadsheets and designates the data corresponding to the behavior of the objects in the simulation. The digital twin is inter-connected in real-time with the actual physical system during its working for behavior analysis and performance optimization. Still, human effort is needed to simulate the problem, and it is checked before the practical implementation of the assembly process. 
After creating the digital twin of the cobot in the Tecnomatix, the next phase is the robot's programming. It still requires substantial time compared to traditional industrial robots. Cobots have much easier programming interfaces, but it still needs substantial time (2 to 3 days), which is not practical to industrial applications. The digital twin is used to create robot program (Figure 19) codes as per the defined assembly sequence and identified LEGO bricks. Once the programing is generated in the digital twin environment, it is tested in the virtual space and after this, the programing is transferred to the real robot to start the assembly process of the LEGO pieces.

\subsection{Application of Study}

Up to know the robots used in industries for different purposes and industries are producing batches of production. And for the changeover, the robot has to be programmed again according to the new components, which a lot of time and human effort. This study can be much helpful for industrial applications like designing, process optimization, and assembly. Due to intelligent digital twin, the robots will become more powerful in decision-taking for different tasks, with this the integration of robots in the industry will become fast. In the case of intelligent digital twins, less amount of programming will be needed, especially in collaborative robots. The study will enable to produce verity-oriented production. The learnings and techniques of this study can be extended to the future study of the safety of humans when working with robots, as this study also shows that due to the safety of humans' cobots run at slow speed, and because of this, it's taking more time for performing the task than the manual process.

\section{CONCLUSION AND FUTURE WORK}

After doing work on this study, I was able to achieve my objectives. I trained and obtained a high accuracy model for the classification of Lego pieces which is almost 96 percent. It was possible because of only choosing the right hyper-parameters and the accurate architecture of the model.

In the wake of making the model and getting the outcomes, the model can be adjusted by changing the number of layers, hyper-boundaries of the layers, thick layer, and the dropout. By altering the model, the model can all the more likely be prepared on account of these better outcomes and precision can be accomplished.

However, the degree of the economic and demographic element might differ from the factor of achievement. In this example, twin model simulation, deep learning, and AI resulted in a better variance [18-
19]. By expanding the information or classes in the informational index the utilization of the model can be augmented or expanded. With this, it very well may be used can motivations behind reenactment, so machines figure out how to perceive the bits of Lego pieces.

One thought is that we can utilize move learning. Using a pre-prepared AI model is called transfer learning. The hidden thought behind transfer learning is that one takes an all-around prepared model from one informational collection or space and applies it to another one.

By using simulation in the Tecnomatix software, I developed the digital twin of the collaborative robot. The CNN model of classification is programmed into the software, by doing this I was able to develop the cobot paths. According to task, programming may take

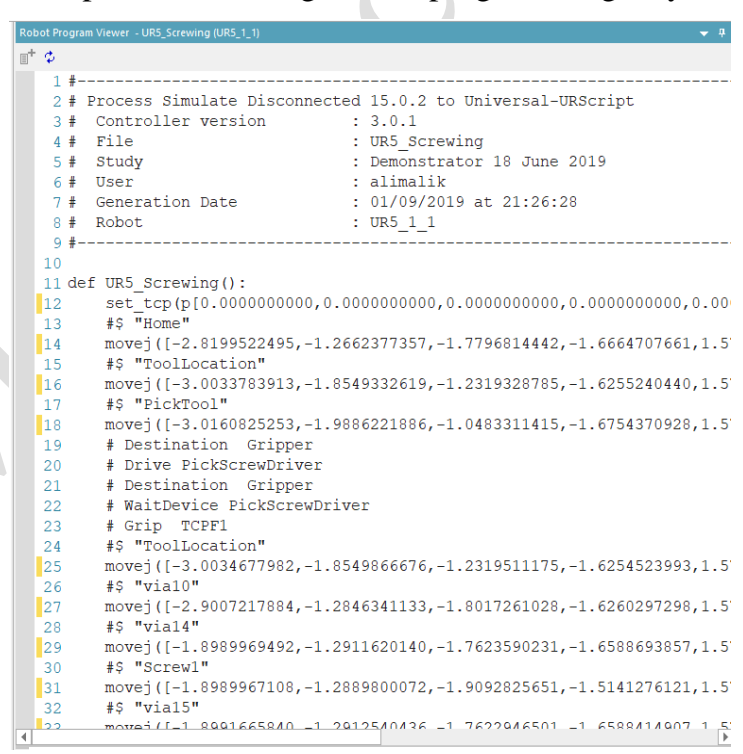

Figure 9: Robot program generated form the digital twin

days or weeks after completing the programing, which was time-consuming and tedious work [20-21]. I tested the programing in the virtual space and the digital twin of the cobot started the assembly process of Lego pieces. After successful testing of the digital twin, information of the digital twin is transferred to the physical or tangible cobot to start the assembly. Thus I was able to make digital twin intelligent with the help of deep learning. 


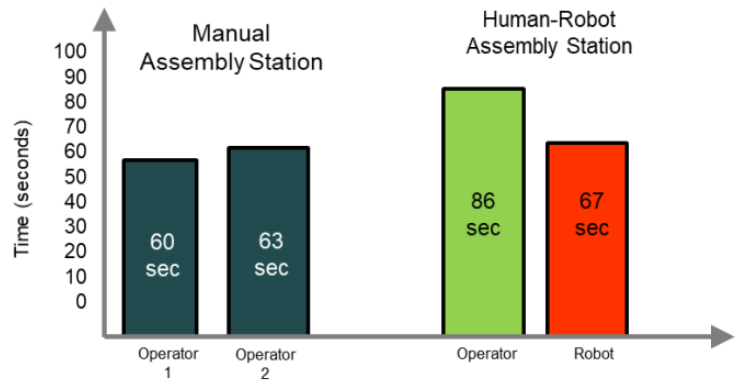

Figure 10: Time consumed by cobot as compared to manual assembly

During the assembly of Lego pieces by cobot, it is observed that the robot is taking more time than the manual assembly process. It is only because the robot is operated at a slow speed for the safety of humans. And it is also a delimitation and future research option to how to make robots safe for humans and thus operate robots at high speed.

\section{REFERENCES}

[1] M. Fera, R. Macchiaroli, F. Fruggiero, and A. Lambiase, "A modified tabu search algorithm for the single-machine scheduling problem using additive manufacturing technology," Int. J. Ind. Eng. Comput., vol. 11, no. 3, pp. 401-414, 2020, doi: 10.5267/j.ijiec.2020.1.001.

[2] A. A. Malik, T. Masood, and A. Bilberg, "Virtual reality in manufacturing: immersive and collaborative artificial-reality in design of human-robot workspace," Int. J. Comput. Integr. Manuf., vol. 33, no. 1, pp. 2237, Jan. 2020, doi: 10.1080/0951192X.2019.1690685.

[3] A. A. Malik and A. Bilberg, "Digital twins of human-robot collaboration in a production setting," Procedia Manuf., vol. 17, pp. 278-285, 2018, doi: 10.1016/j.promfg.2018.10.047.

[4] Yaseen, Asif. "Next-Wave of E-commerce: Mobile Customers Churn Prediction using Machine Learning." LGURJCSIT, vol 5, issue2, pp. 62-72, 2021."

[5] Malik, Ali Ahmad, Tariq Masood, and Rehana Kousar. "Reconfiguring and ramping-up ventilator production in the face of COVID-19: Can robots help?." Journal of Manufacturing Systems, 60, 864875, 2021.

[6] B. Schleich, N. Anwer, L. Mathieu, and S. Wartzack, "Shaping the digital twin for design and production engineering," CIRP Ann. - Manuf.
Technol., vol. 66, no. 1, pp. 141-144, Jan. 2017, doi: 10.1016/j.cirp.2017.04.040.

[7] F. Juefei-Xu, V. N. Boddeti, and M. Savvides, "Local binary convolutional neural networks," Proc. 30th IEEE Conf. Comput. Vis. Pattern Recognition, CVPR 2017, vol. 2017-Janua, pp. 4284-4293, 2017, doi: 10.1109/CVPR.2017.456.

[8] Y. Wang, C. Xu, C. Xu, C. Xu, and D. Tao, "Learning versatile filters for efficient convolutional neural networks," Adv. Neural Inf. Process. System., vol. 2018-Decem, no. NeurIPS, pp. 1608-1618, 2018.

[9] K. He, X. Zhang, S. Ren, and J. Sun, "Deep residual learning for image recognition," Proc. IEEE Comput. Soc. Conf. Comput. Vis. Pattern Recognition., vol. 2016-Decem, pp. 770-778, 2016, doi: 10.1109/CVPR.2016.90.

[10] X. Gao, Y. Zhao, L. Dudziak, R. Mullins, and X. Cheng-Zhong, "Dynamic channel pruning: Feature boosting and suppression," 7th Int. Conf. Learn. Represent. ICLR 2019, pp. 1-14, 2019.

[11] Lin, Keng-Pei, and Ming-Syan Chen. "On the design and analysis of the privacy-preserving SVM classifier." IEEE transactions on knowledge and data engineering, vol 23, issue 11,1704-1717, 2010.

[12] Edward, J., Wannasuphoprasit, C.W. and Peshkin, M.A. Cobots: Robots for collaboration with human operators. In International Mechanical Engineering Congress and Exposition. pp. 1115,1996 .

[13] Djuric, A.M., Urbanic, R.J. and Rickli, J.L. A framework for collaborative robot (CoBot) integration in advanced manufacturing systems. $S A E$ International Journal of Materials and Manufacturing, vol 9, issue 2, pp.457-464, 2016.

[14] Verdouw CN, Kruize JW."Digital twins in farm management: illustrations from the FIWARE accelerators SmartAgriFood and Fractals." In Proceedings of the 7th Asian-Australasian Conference on Precision Agriculture Digital, Hamilton, New Zealand, pp. 16-18. 2017.

[15] D. Mourtzis, A. Vasilakopoulos, E. Zervas, and N. Boli, "Manufacturing System Design using Simulation in Metal Industry towards Education 4.0," Procedia Manuf., vol. 31, pp. 155-161, 2019, doi: 10.1016/j.promfg.2019.03.024. 
[16] Wilhelm, Jasper, Thies Beinke, and Michael Freitag. "Improving Human-Machine Interaction with a Digital Twin." In International Conference on Dynamics in Logistics, pp. 527-540. Springer, Cham, 2020.

[17] Nof, S.Y., Wilhelm, W.E. \& Warnecke, HIndustrial assembly, Springer Science \& Business Media, 2012.

[18] Christine ZEH Simon BABOVIC. Lego Recognition Tool. 1.4 Technologies, vol 1, issue 1,2017

[19] Lechler, Tobias, Eva Fischer, Maximilian Metzner, Andreas Mayr, and Jörg Franke. "Virtual Commissioning-Scientific review and exploratory use cases in advanced production systems." Procedia CIRP vol 81,pp. 1125-1130, 2019.

[20] Zhang, M., Zuo, Y. and Tao, F., March. Equipment energy consumption management in digital twin shop-floor: A framework and potential applications. In 2018 IEEE 15th International Conference on Networking, Sensing and Control (ICNSC), pp. 1-5, 2018.

[21] Z. Zahid, M. U. Sattar, H. W. Khan, A. Zahid, and M. F. Riaz, "A Smart Analysis and Visualization of the Power Forecasting in Pakistan,", International Journal of Computing and Digital Systems. Vol 10, 2007. 